\title{
ENDOMORPHISMS OF FIBRED GROUPS
}

\author{
by CARLTON J. MAXSON and GUNTER F. PILZ
}

(Received 7th July 1987)

A collection $\mathscr{F}=\left\{G_{\alpha} \mid \alpha \in A\right\}$ of proper subgroups $G_{\alpha}$ of a group $G$ is a fibration of $G$ if

$$
G=\bigcup_{\alpha \in A} G_{\alpha}, \quad G_{\alpha} \cap G_{\beta}=\{1\} \quad \text { for } \quad \alpha \neq \beta
$$

It is of geometric interest to associate two semigroups to a group $G$ with fibration $\mathscr{F}$ :

$$
\begin{aligned}
& E:=E(G, \mathscr{F}):=\left\{h \in \operatorname{End} G \mid h\left(G_{\alpha}\right) \subseteq G_{\alpha} \text { for all } \alpha \in A\right\} \\
& S:=S(G, \mathscr{F}):=\left\{h \in \text { End } G \mid \text { for each } \alpha \in A \text { there is some } \beta \in A \text { with } h\left(G_{\alpha}\right) \subseteq G_{\beta}\right\} .
\end{aligned}
$$

The elements of $E$ are dilatations of the associated translation plane, while the elements of $S$ are endomorphisms of $G$ which are at the same time operators for this translation plane (for more details on this, see e.g. [6]).

All groups are finite. Clearly, $E \subseteq S$ always holds.

Theorem. For each finite fibred group $G, E \neq S$.

Proof. The proof requires several steps. We assume $E=S$.

(1) Suppose $G$ has a non-trivial centre $Z(G)$, By [3, p. 199], either $G$ has a prime exponent $p$ or $Z(G)$ is contained in a single fibre $G_{0}$ (say). In the first case, $G$ is nilpotent and hence has a maximal normal subgroup of index $p$. In the second case $Z(G)$ has prime exponent $p[1$, Bemerkung, 2.4] and all elements of $G$ of order $\neq p$ are contained in $G_{0}$ [1, Lemma 2.1]. If $N$ is the subgroup generated by $Z(G)$ and all elements of order $\neq p$ then $N$ is a subgroup of $G_{0}$. Since conjugation preserves order, it is routine to check that $N$ is a normal subgroup of $G$, contained in $G_{0}$. Hence $G / N$ is a $p$-group and by the homomorphism theorem we again get a maximal normal subgroup of index $p$ in $G$.

(2) If $N$ is a normal subgroup of $G$ of prime index, we have $G / N \cong Z_{p}$. Let $g \in G \backslash N$ be of order $p$. Then $G / N \cong\langle g\rangle$; so we get an endomorphism $h \neq i d$ of $G$ mapping all of $G$ into the single fibre containing $g$. Hence $h \in S$, but $h \notin E$, a contradiction. 
(3) Hence we can assume that $Z(G)=\{1\}$. If $E \neq\{0$,id $\}$, Theorem II.3 of [7] implies that $G$ must have a non-trivial centre $(0$ denotes the trivial endomorphism). Hence we are down to the case $E=S=\{0, i d\}, Z(G)=\{1\}$.

(4) Suppose that the Fitting subgroup $F G$ of $G$ is trivial. By [5] or [9] $G$ must fall into one of the following classes:

(i) $G \cong P G L\left(2, p^{n}\right), p^{n} \geqq 4$

(ii) $G \cong P S L\left(2, p^{n}\right), p^{n} \geqq 4$

(iii) $G$ is a simple Suzuki group.

Recall that by [9, Lemma 1], any fibration can be refined into a normal (=kinematic in [3]) one. Since a normal fibration has $\{i d\} \neq \operatorname{Inn} G \subseteq S$, we can exclude these ones. In all cases (i)-(iii), an examination of the proofs of the results of [5] shows that $\mathscr{F}$ arises from such a normal fibration $\mathcal{N}$ by taking the normalizer $N$ of a suitable Sylow subgroup of $G$ and all fibres of $\mathcal{N}$ not contained in $N$. Take some $x \in N, x \neq 1$. Then $x$ determines an inner automorphism $\phi_{x} \neq i d$ of $G$. Since $\phi_{x} \in \operatorname{Inn} G$ and $\mathscr{N}$ is normal, each $\phi_{x}\left(N_{\alpha}\right) \in \mathscr{N}$ for $N_{\alpha} \in \mathscr{N}$. Trivially $\phi_{x}(N)=N$ since $x \in N$. Hence $i d \neq \phi_{x} \in S$, and $F G=\{1\}$ cannot happen.

(5) Finally, we study the case $F G \neq\{1\}$. From [2] and [4], either

(i) $G$ is a $p$-group, or

(ii) $G$ is a Frobenius group, or

(iii) $G \cong S_{4}$, or

(iv) $Z(G) \neq\{1\}$.

Now (i) and (iv) are excluded by (1) and (2).

In case (ii), we study a normal refinement $\mathscr{F}^{*}$ (see [8]) of $\mathscr{F}$. By [1, Satz 4.1], $\mathscr{F}^{*}$ consists of subgroups $G_{i}(i \in I)$ of $F G$ and of subgroups $G_{j}(j \in J)$ which are selfnormalizing. By Satz 4.7 of [1], $\mathscr{F} *$ consists of some (possibly different) subgroups $G_{k}(k \in K)$ of $F G$ and the same subgroups $G_{j}$ as above. Since $\mathscr{F}$ is normal, for each inner automorphism $\phi_{x}$ induced by $x \in G, \phi_{x}\left(G_{j}\right)$ is some $G_{j^{\prime}}\left(j, j^{\prime} \in J\right)$.

$F G$ is nilpotent and hence has a non-trivial centre. Take $z \neq 1$ in the centre. Then $\phi_{z}=i d$ on $F G$, but $\phi_{z}\left(G_{j}\right)=G_{j}$ is impossible for $j \in J$, since each $G_{j}$ coincides with its normalizer. Hence $\phi_{z}$ is in $S$, but not in $E$.

Finally, let $G \cong S_{4}$. In this case, $A_{4}$ is normal of prime index. We can proceed as in (2) to get some $h \in S \backslash E$, and we are done.

Corollary 1. A finite group cannot have a fibration of fully invariant subgroups.

Now we write $G$ additively (this does not imply commutativity). It is also of geometric interest (see [7]) to consider the collection of all possible sums $d g E$ of elements of $E=E(G, \mathscr{F}) . E$ is a distributively generated near-ring (see e.g. [8]). The same applies to $\operatorname{dg} S$. Clearly, $\operatorname{dg} E$ is a subnear-ring of $\operatorname{dg} S$. If $\operatorname{dg} E=\operatorname{dg} S$, each $s \in S \subseteq d g S$ 
must map each cell into itself, because every sum in $\operatorname{dg} E$ behaves that way. Hence we have the following.

Corollary 2. If $G$ is a finite fibred group then $d g E \neq d g S$.

\section{REFERENCES}

1. R. BAER, Partitionen einfacher Gruppen, Math. Z. 75 (1961), 333-372.

2. R. BaER, Einfache Partitionen endlicher Gruppen mit nicht-trivialer Fitting'scher Untergruppe, Arch. Math. 11 (1961), 81-89.

3. H. Karzel and C. J. Maxson, Fibered groups with non-trivial centers, Results in Math. 7 (1984), 192-208.

4. O. H. Kegel, Nicht-einfache Partitionen endlicher Gruppen, Arch. Math. 12 (1961), $170-175$.

5. O. H. Kegel, Aufzählung der Partitionen endlicher Gruppen mit trivialen Fitting'schen Untergruppen, Arch. Math. 12 (1961), 409-412.

6. C. J. Maxson, Near-rings associated with generalized translation structures, J. Geom. 24 (1985), 175-193.

7. C. J. Maxson and G. Pilz, Near-rings determined by fibered groups, Arch. Math. 44 (1985), 311-318.

8. G. PILz, Near-rings, $2^{\text {nd }}$ ed. (North-Holland, Amsterdam-New York, 1983).

9. M. Suzukı, On a finite group with a partition, Arch. Math. 12 (1961), 241-254.

Department of Mathematics

TEXAS A\&M UNIVERSITY

College Station, TX 77843

U.S.A.
INSTITUT FÜR MATHEMATIK JohanNes Kepler Universität Linz 4040 LiNZ Austria 